\title{
Welcome to volume 10 of Bioanalysis
}

\author{
Sankeetha Nadarajah*,1 \& Hannah Makin ${ }^{1}$ \\ ${ }^{1}$ Future Science Group, Unitec House, 2 Albert Place, London N31QB, UK \\ * Author for correspondence: s.nadarajah@future-science.com
}

First draft submitted: 13 November 2017; Accepted for publication: 13 November 2017; Published online: 19 December 2017

Keywords: bioanalysis $\bullet$ publications $\bullet$ research

Welcome to volume 10 of Bioanalysis. We want to take this opportunity to wish you all a Happy New Year. As we step into the tenth year of the journal, we would like to reflect on the past 12 months from the journal's perspective. We want to thank all our authors, readers and reviewers, as well as our Editorial Board members for their continued support. We very much look forward to working with everyone in 2018.

\section{Content highlights}

Bioanalysis publishes a variety of articles covering key advances in this ever-evolving field and this section looks at some of the most read content of the year, at the time of writing this article (November 2017).

Similar to last year, we saw several meeting reports featuring in our top read content. Our most read article was the Conference Report from the 10th GCC closed forum [1], the objective of which was for CRO bioanalytical representatives to meet and discuss scientific and regulatory issues specific to bioanalysis. Feedback from the European Bioanalysis Forum on the Focus Workshop on 'Bringing assay validation and analysis of biomarkers into practice' was also one of our most read reports [2], as was the one from the 37th British Mass Spectrometry Society annual meeting [3].

Recommendation papers continued to be popular among the readers in 2017. 'Best practices in performing flow cytometry in a regulated environment: feedback from experience within the European Bioanalysis Forum' was one of our most well-read White Papers [4], while 'Recommendations for clinical biomarker specimen preservation and stability assessments', by Naveen Dakappagari, Hui Zhang, Laurie Stephen, Lakshmi Amaravadi and Masood U Khan, have also been highly accessed [5]. We have also recently published a special feature on'The Decennial Index of the White Papers in Bioanalysis: A Decade of Recommendations (2007-2016)' [6], which is already reaching a wide audience. After 10 years of publishing White Papers summarizing the conclusions and consensus points from each Workshop on Recent Issues in Bioanalysis meeting, in which a wide range of industry opinion leaders and regulatory authorities come together, the recent publication acts as a general index organized by topic to easily consult all these recommendations and their evolution over time.

Several editorial-style articles providing a snapshot of issues of topical importance to the bioanalytical community also featured in our most read content. Several novel approaches were explored this year, including 'Rapid prototyping using 3D printing in bioanalytical research' [7] in an editorial by Chengsen Zhang, Brandon J Bills and Nicholas E Manicke, while the unique opportunities of 'Paper-based point-of-care testing in disease diagnostics' were looked at by Leila Syedmoradi and Frank A Gomez [8].

We publish a range of research-style articles presenting novel work and representing important advancements in the understanding of techniques, which continue to be highly accessed. A couple of these included a Research article on the "Development and validation of $L C-M S / M S$ with in-source collision-induced dissociation for the quantification of pegcantratinib in human skin tumors' [9] and a methodology looking at the 'Implementation of a novel ultra-fast metabolic stability analysis method using exact mass TOF-MS' [10]. A Perspective article on the practical approach to 'Demonstrating biosimilar and originator antidrug antibody-binding comparability in antidrug antibody assays' [11] and a piece looking at the impact of 'Integrating ion mobility spectrometry into mass spectrometry-based exposome measurements' [12] were some of the most viewed review-style pieces, which aim to highlight recent significant advances in research, ongoing challenges and unmet needs in which authors provide a concise and critical appraisal of the subject matter. 
Table 1. Special focus/themed issues published in 2016

\begin{tabular}{|c|c|c|c|}
\hline Topic & Guest editor(s) & Issue & Ref. \\
\hline $\begin{array}{l}\text { Methods and techniques for metabolic } \\
\text { phenotyping }\end{array}$ & Ian Wilson (Imperial College, UK) & Vol. 9; No. 1 & [13] \\
\hline Outsourcing strategies in bioanalysis & $\begin{array}{l}\text { Neil Spooner (Spooner Bioanalytical Solutions, UK); Stephanie Cape } \\
\text { (Covance, USA) and Scott Summerfield (GlaxoSmithKline, UK) }\end{array}$ & Vol. 9; No. 15 & [14] \\
\hline Bioanalysis of biopharmaceuticals & $\begin{array}{l}\text { Yan J Zhang (Bristol-Myers Squibb, NJ, USA), Hyun Joo An (Chungnam } \\
\text { National University, Daejeon, Korea) }\end{array}$ & Vol. 9; No. 18 & [15] \\
\hline Emerging technologies in MS & $\begin{array}{l}\text { Larry Campbell (University of Waterloo, Canada), Robert Plumb (Waters } \\
\text { Corporation, USA) }\end{array}$ & Vol. 9; No. 21 & [16] \\
\hline
\end{tabular}

As we head into 2018, we will endeavor to continue capturing in the journal the trends and evolution of the field of bioanalysis.

\section{Special focus issues}

The aim of our special focus issues is to highlight happening themes in the field of bioanalysis. 2017 saw a range of topics for which we wanted to initiate a greater level of interest and awareness of the topics among the broader audience, and the details of these issues can be seen in Table 1. The year kicked off with a focused issue on 'Methods \& Techniques for Metabolic Phenotyping', aiming to highlight current applications and advances in the field of metabolic phenotyping, while also providing an outlook on how the field is likely to evolve [13]. 'Outsourcing Strategies in Bioanalysis' was the focus of the second themed issue of the year, in which models and trends in outsourcing were looked at from multiple angles, collating the experiences of various experts in the industry [14]. Then, the challenges, advancements and novel strategies in the Bioanalysis of Biopharmaceuticals were discussed in detail [15]. In the final themed issue of the year, several emerging technologies in MS that could in the near future provide dramatic changes to what is considered conventional bioanalysis were shared [16].

Each of our special focus issues included an introductory Foreword article by the Guest Editors of the respective issues and these were among the most accessed content of the year as were several of the content pieces. It is encouraging to see that we are capturing some of the current hot topics of the industry and we continue to keep doing so, with a number of exciting themed issues in progress for 2018. These will include focuses on the topics of: Qualification and Validation of Biomarkers; Protein Therapeutics \& Target Quantification by LC-MS; Novel LC-MS Assays Impacting CYP \& Transporter DDI Evaluations and Bioanalytical Techniques in Determining Immunogenicity, among other themes.

\section{Bioanalysis in the community}

The key aim of the Bioanalysis editorial team is to ensure that the journal remains focused on the key themes and trends in the bioanalytical community, and we strive to achieve a strong presence in this community through reaching out to our readers and contributors. Our editors often attend relevant symposiums and conferences across the world as representatives of the journal, establishing networks and stable working relations with experts in the area and keeping up to date with the most recent advances in this fast-evolving field.

We welcome unsolicited manuscript submissions through the online manuscript processing portal ScholarOne Manuscripts ${ }^{\mathrm{TM}}{ }_{[17]}$, and are also happy for authors to contact our editorial team with feedback, suggestions, ideas and proposals for articles. It is also noteworthy that Future Science partners with Enago [18] to provide presubmission editing services for our authors.

We are on social media, where we continue to post about our articles through a Bioanalysis LinkedIn group [19], providing followers with the latest journal news and updates and a Twitter account (@fsgbio) [20] that provides updates on the latest and most interesting bioanalysis news and developments from the global community.

Higher readership is consistently seen by our articles that have been shared by the authors, so we are pleased to continue our partnership with Kudos [21] in the New Year, to support our authors in sharing their work and tracking the outcomes of their activities. As social media evolves to become a powerful tool in disseminating scientific publications, we now also work with Altmetrics to measure the impact of our publications [22].

\section{Demographics of readers \& contributors}

Bioanalysis has a wide reach, with both subscribers and authors based all around the globe. There has been an increase in the number of subscribers to the journal that are from Europe this year, and we continue to see growth 
(A)

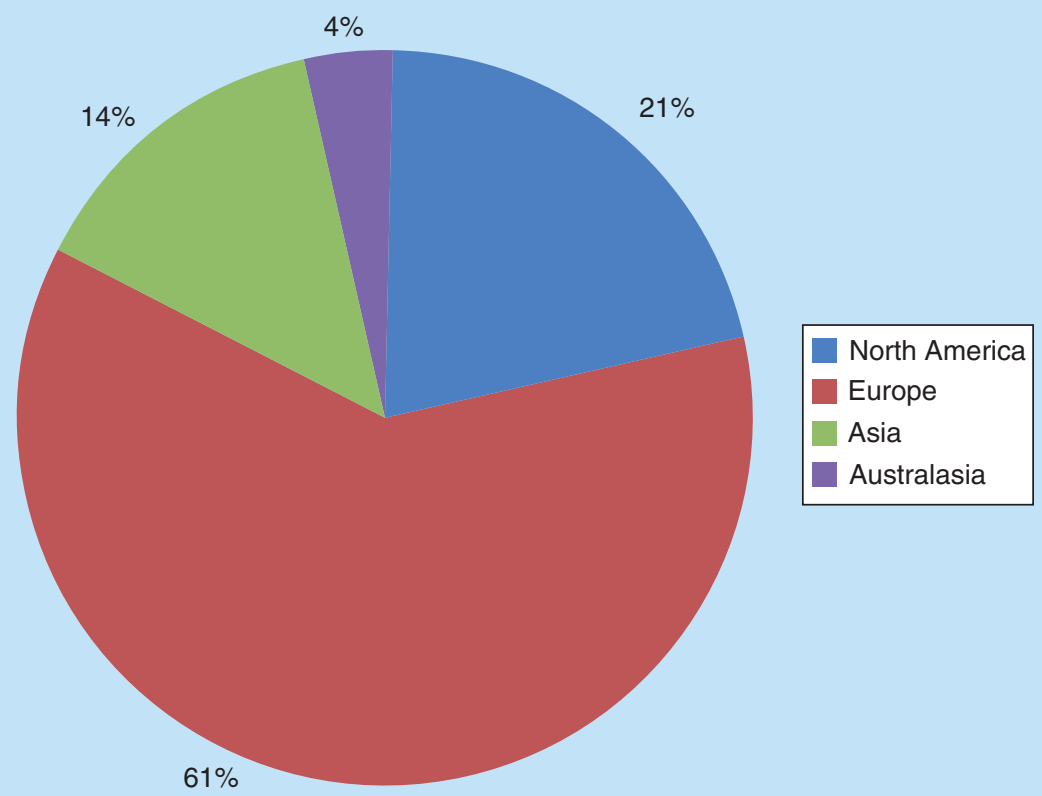

(B)

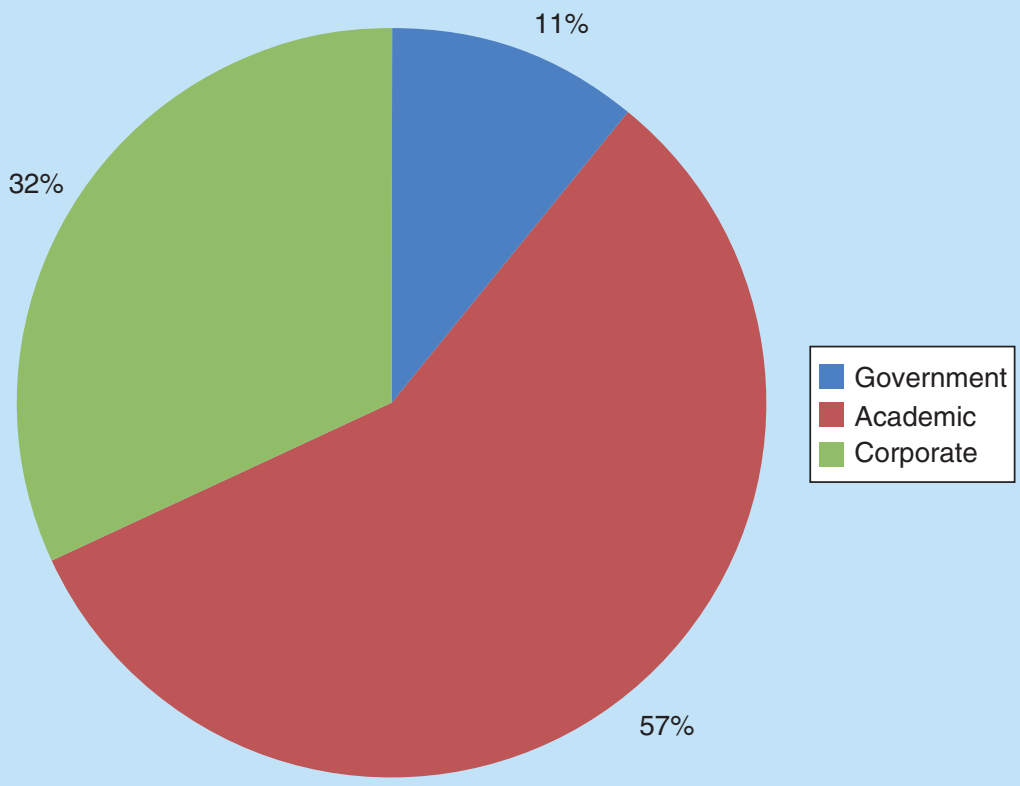

Figure 1. Readership 2017.

Percentage of subscriptions by (A) geographic location and (B) sector.

in the number of subscriptions from Asia and the rest of the world, as shown in (Figure 1A). The journal also attracts readers from a variety of working environments (Figure 1B) as we strive to work with experts from across both corporate and academic surroundings. Bioanalysis receives contributions from authors from across the globe (Figure 2), and the spread appears similar to that seen in 2016, 


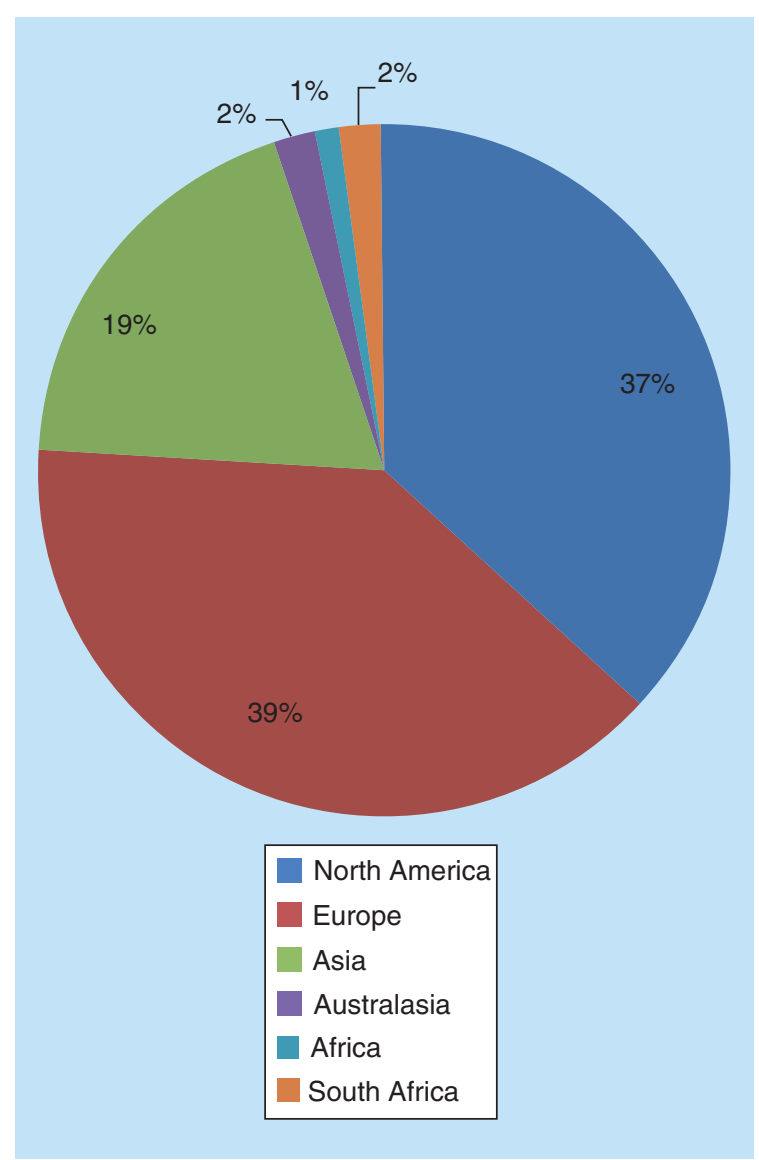

Figure 2. Authorship 2017.

\section{Bioanalysis zone}

If you have not yet visited Bioanalysis zone [23], we highly recommend you to take a look around the site, which is an interactive online resource for the bioanalytical community. Since launching in 2011, Bioanalysis zone has attracted over 9500 active members from the global bioanalytical community, representing the pharmaceutical, biotech and CRO industries, along with academia and healthcare. Each month, thousands of members visit the site to read industry news, original research exclusive interviews and commentaries. It provides a forum for the community to discuss recent developments and pose any questions related to this fast-moving field, and membership is completely free of charge.

The Journal and the zone work in collaboration on several projects, with the vision of ensuring research and advancements in the field are presented in the most appropriate and accessible manner to the bioanalytical community. One of the successful collaborations of 2017 included the publication of the Panel Discussions that we organized together, in which experts from leading CROs, consultancies and pharmaceutical companies conversed about the 'Changing World of Bioanalysis'. The Panel Discussion Report was one of our top read articles of the year [24], while the videos of the discussions remain some of the most viewed content on the site [25].

\section{Conclusion}

We appreciate the feedback that has been provided over the last year as to the direction of the journal, and look forward to more in the year to come. Finally, we would like to thank everyone for your continued engagement and support, and we look forward to working with all of you over the coming year. 


\section{References}

1 Cape S, Islam R, Nehls C et al. The 10th GCC Closed Forum: rejected data, GCP in bioanalysis, extract stability, BAV, processed batch acceptance, matrix stability, critical reagents, ELN and data integrity and counteracting fraud. Bioanalysis 9(7), 505-516 (2017).

2 Timmerman P, Scheel Fjording M, Allinson J et al. Feedback from the EBF - focus workshop: bringing assay validation and analysis of biomarkers into practice. Bioanalysis 9(9), 675-681 (2017).

3 Wright P, Eckers C. 37th British Mass Spectrometry Society annual meeting. Bioanalysis 9(5), 423-426 (2017).

4 der Strate BV, Longdin R, Geerlings $\mathrm{M}$ et al. Best practices in performing flow cytometry in a regulated environment: feedback from experience within the European Bioanalysis Forum. Bioanalysis 9(16), 1253-1264 (2017).

5 Dakappagari N, Zhang H, Stephen L, Amaravadi L, Khan MU. Recommendations for clinical biomarker specimen preservation and stability assessments. Bioanalysis 9(8), 643-653 (2017).

6 Garofolo W, Savoie N. The decennial index of the White Papers in bioanalysis: 'a decade of recommendations (2007-2016). Bioanalysis doi:10.4155/bio-2017-4979 (2017) (Epub Ahead of Print).

7 Zhang C, Bills BJ, Manicke NE. Rapid prototyping using 3D printing in bioanalytical research. Bioanalysis 9(4), 329-331 (2017).

8 Syedmoradi L, Gomez FA. Paper-based point-of-care testing in disease diagnostics. Bioanalysis 9(11), 841-843 (2017).

9 Zangarini M, Rajan N, Danilenko M, Berry P, Traversa S, Veal GJ. Development and validation of LC-MS/MS with in-source collision-induced dissociation for the quantification of pegcantratinib in human skin tumors. Bioanalysis 9(3), 279-288 (2017).

10 Manna JD, Richardson SJ, Moghaddam MF. Implementation of a novel ultra fast metabolic stability analysis method using exact mass TOF-MS. Bioanalysis 9(4), 359-368 (2017).

11 Ryding J, Stahl M, Ullmann M. Demonstrating biosimilar and originator antidrug antibody binding comparability in antidrug antibody assays: a practical approach. Bioanalysis 9(18), 1395-1406 (2017).

12 Metz TO, Baker ES, Schymanski EL et al. Integrating ion mobility spectrometry into mass spectrometry-based exposome measurements: what can it add and how far can it go? Bioanalysis 9(1), 81-98 (2017).

13 Methods \& techniques for metabolic phenotyping. www.future-science.com/toc/bio/9/1

14 Outsourcing strategies in bioanalysis. www.future-science.com/toc/bio/9/15

15 Bioanalysis of biopharmaceuticals. www.future-science.com/toc/bio/9/18

16 Emerging technologies in MS. www.future-science.com/toc/bio/9/21

17 ScholarOne Manuscripts ${ }^{\mathrm{TM}}$ - Bioanalysis. https://mc04.manuscriptcentral.com/fs-bio

18 Future Science - Enago. http://futurescience.enago.com/

19 Bioanalysis LinkedIn Group. www.linkedin.com/groups/2819540.

20 Bioanalysis Twitter Page. https://twitter.com/fsgbio

21 Future Science - Kudos. http://www.future-science.com/page/kudos

22 Altmetrics. www.altmetric.com

23 Bioanalysis Zone. www.bioanalysis-zone.com

24 Spooner N, Anderson M, Dillen L et al. The changing world of bioanalysis: summary of panel discussions. Bioanalysis 9(15), 1175-1179 (2017).

25 Bioanalysis Zone: The changing world of bioanalysis panel discussion. www.bioanalysis-zone.com/2017/02/01/cwb_spo/ 
